\title{
OCHRONOSIS- A CASE REPORT
}

Kanika Bansal', Rakesh Kakkar²

1 Medical Student, Department of Pathology, Bharti Vidyapeeth (Deemed to be University), Pune, Maharashtra, India.

2Professor and Senior Consultant, Department of Pathology, VIMS, Gajraula, Moradabad, Uttar Pradesh, India.

HOW TO CITE THIS ARTICLE: Bansal K, Kakkar R. Ochronosis- a case report. J. Evolution Med. Dent. Sci. 2018;7(22):2723-2725, DOI: $10.14260 /$ jemds/2018/614

\section{PRESENTATION OF CASE}

Connective tissue is generally affected in ochronosis. Recognition of changes secondary to the deposition of ochronotic pigments has increased with advances in diagnostic technology, allowing both improved imaging and early biochemical and genetics-based diagnosis of alkaptonuria, the cause of ochronosis. Successful symptomatic treatment of ochronotic arthropathy with joint replacement has been documented and a new pharmacotherapeutic agent, Nitisinone is currently used for both prevention and treatment of ochronosis.

A 53 years old male came with the complaints of pain and stiffness in both knee joint and lower back pain for the past 3 years. He was intermittently treated with analgesics without total relief. There was gradual deterioration of the symptoms in the knee joint with restricted movements, which forced him to report to an orthopaedic surgeon. Later on, was taken for bilateral meniscectomy. After surgery, was put on palliative treatment and drug Nitisinone was advised.

\section{On examination, the following findings were seen:}

1. Grey-blue or blue-black patches of skin, typically on the cheek and lower aspect of back.

2. The skin lesions are present for about more than 3 years.

3. Joint inflammation and stiffness, mainly in knee and back.

\section{DIFFERENTIAL DIAGNOSES}

1. Alkaptonuria.

2. Ochronosis.

3. Osteoarthritis.

4. Ankylosing spondylitis.

5. Paget's disease of the bone.

'Financial or Other Competing Interest': None.

Submission 28-01-2018, Peer Review 14-05-2018,

Acceptance 21-05-2018, Published 28-05-2018.

Corresponding Author:

Kanika Bansal,

D-1501, One North,

Behind Seasons Mall,

Pune-411028,

Maharashtra, India.

E-mail: bansalkanika96@gmail.com

DOI: $10.14260 /$ jemds $/ 2018 / 614$

\section{(c) (i) $€$}

\section{CLINICAL DIAGNOSIS}

1. X-ray:

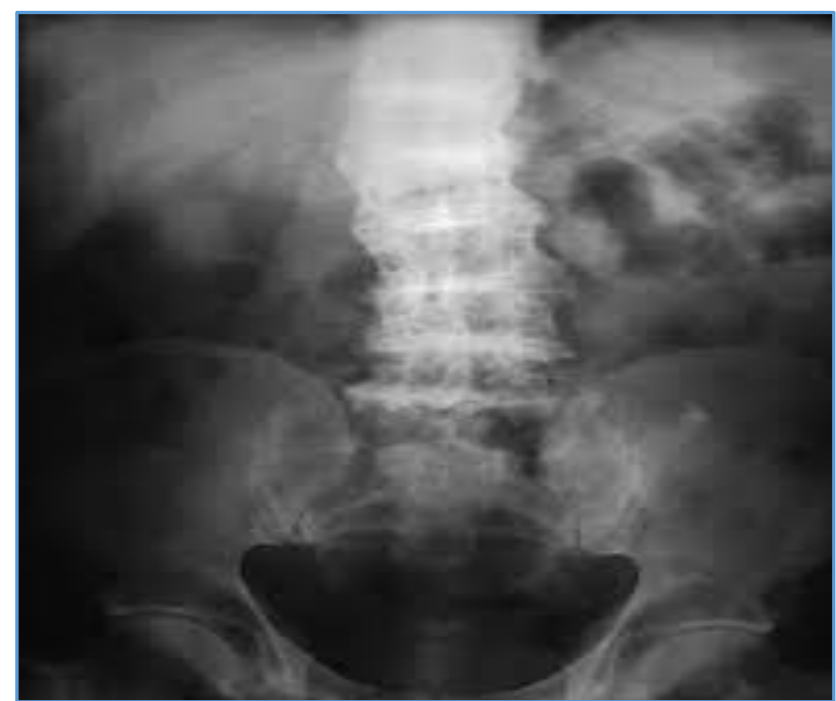

Severe Osteoporosis, Multilevel Intervertebral Disc Calcification, Syndesmophyte Formation, Multilevel Disc Space Narrowing $[1,2,3]$

2. MRI: More accurate and reliable.[4,1,2,3]

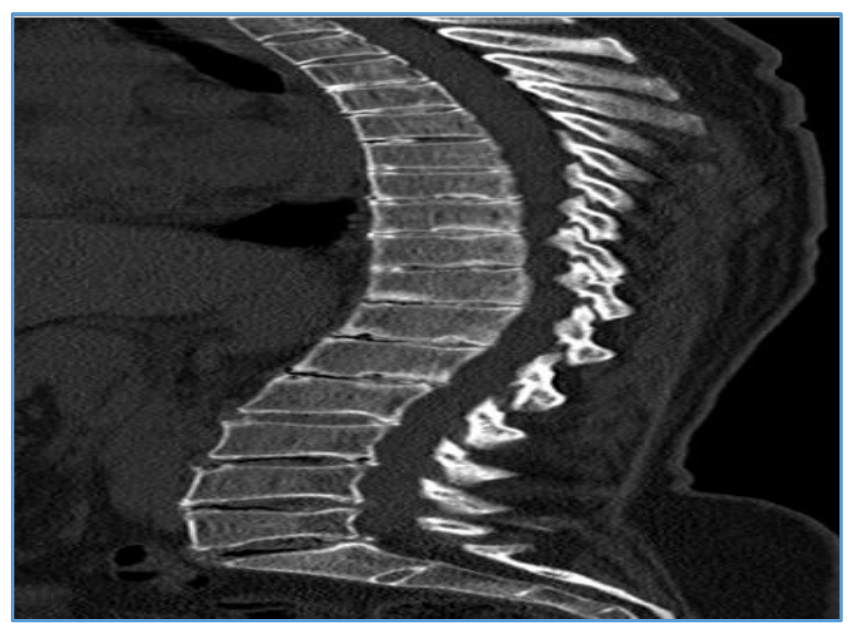

Syndesmophyte Formation, Multilevel Disc Space Narrowing

3. Bone Scintigraphy: Intervertebral discs showed a high uptake which extended laterally from the axial vertebral column; the finding gave an impression of whiskers and this "whisker sign" may be characteristic of ochronosis.[4] 


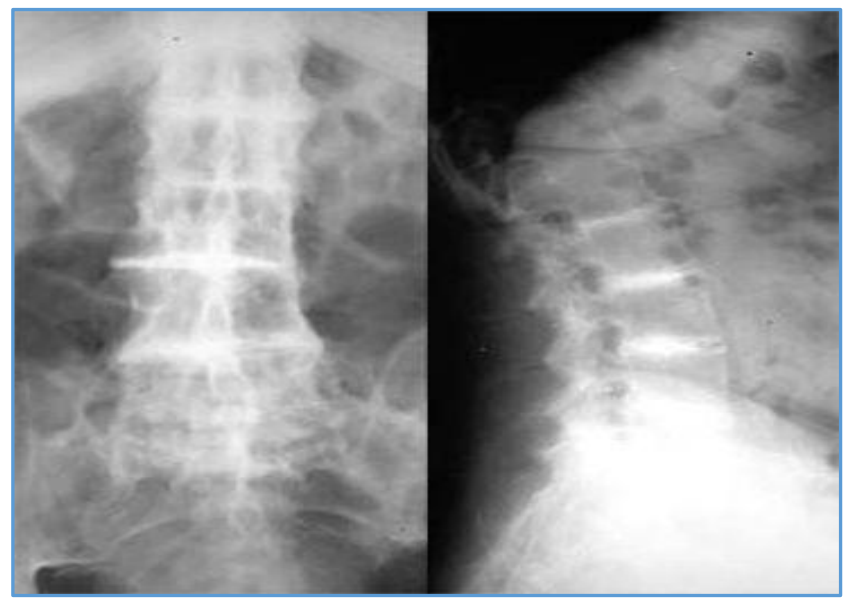

Whisker Sign

4. Ocular Examination: Bluish-black discolouration of sclera present in the eyes. $[5,6,7,4]$

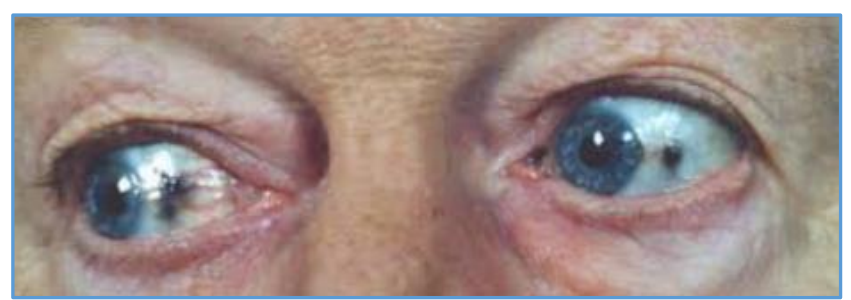

Bluish-Black Discolouration of Sclera

\section{PATHOLOGICAL DIAGNOSES}

1. Skin Biopsy: Samples with haematoxylin and eosin staining reveal yellowish brown-pigmented bodies in the dermis that represent altered widened elastic fibres as well as in macrophages, endothelial cells, apocrine glands and epidermal basement membranes. The deposits do not lose their pigmentation after 3 days in $10 \%$ hydrogen peroxide. Furthermore, the ochronotic pigment reacts with all routine stains for melanin. Such deposits can also be seen in cartilage and elastic tissue. $[5,6,7,4,2]$

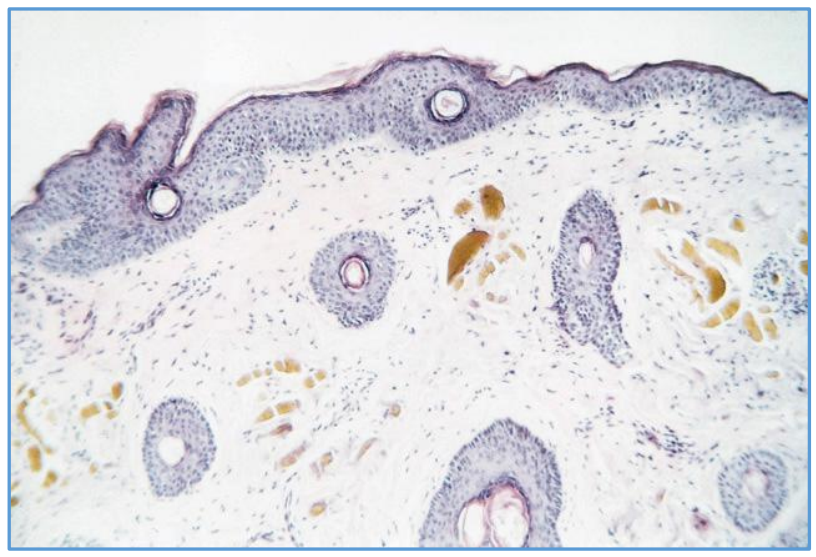

Upon Microscopic Examination, Amber-Coloured, Oval Structures are detected in the Mid-to-Upper Dermal Tissues

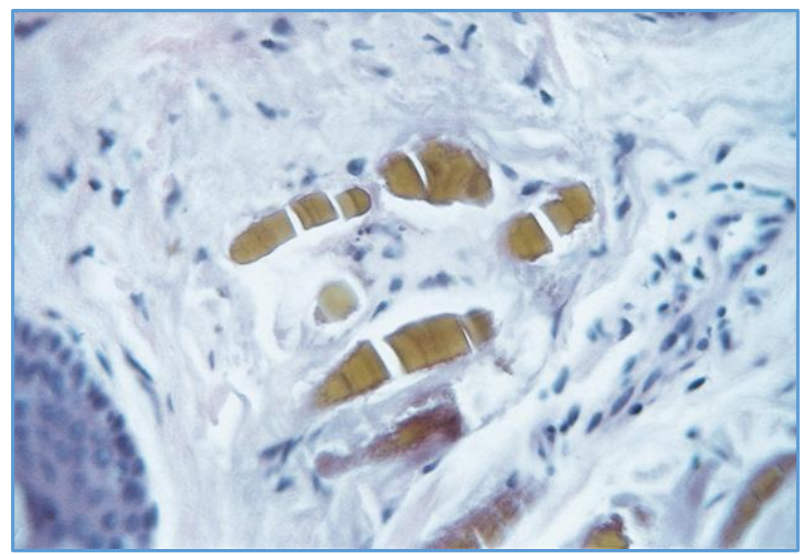

Upon Higher Magnification, Ochronosis reveals Homogenization and Swelling of Collagen Bundles

2. Urine Examination: Blackish-brown colour of urine on contact with air after few hours. [7,4,1,2]

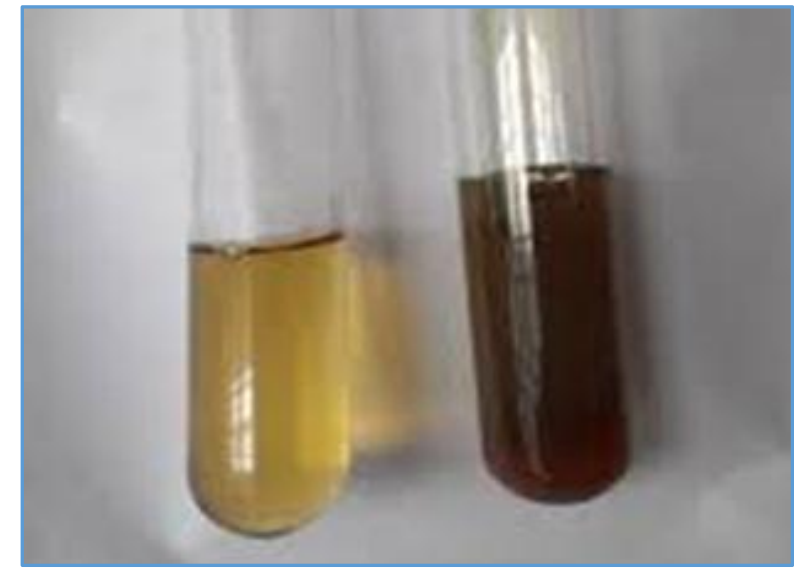

Urine Sample tends to Darken after few hours on Contact with Air

3. Synovial Fluid Examination: Aspiration of synovial fluid revealed some floating black particles. Microscopic examination showed a variety of golden-brown cartilage fragments including those seen in A and B. The osteoarthritis seen in such patients is due to cartilage fragility caused by deposition of polymers of homogentisic acid, which accumulate because of the inherited deficiency of homogentisic acid oxidase.[4]

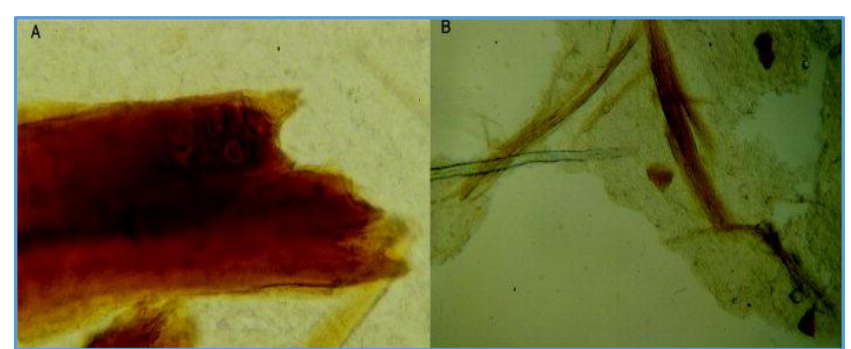

A: Shows Fragment of Pigmented Cartilage with a Few visible Chondrocytes,

B: Shows the more frequent Pigmented Fibrillar Connective Tissue. This Pattern of Particles that appear Black on gross examination and Golden-Brown on Microscopy is Virtually Diagnostic of Alkaptonuria and Ochronosis. 


\section{DISCUSSION OF MANAGEMENT}

\section{A. Medical Care}

- Use of sun protection such as sunscreen, hats, sungoggles and protective clothing can limit damage to the skin from sunlight. $[5,7]$

- Diets low in tyrosine and phenylalanine, thereby reducing the toxic by-product homogentisic acid (HGA).

- Using high doses of vitamin E and C- both vitamins act as depigmenting agents. ${ }^{[5,7]}$

- Use of antioxidants that work well with vitamin E and $\mathrm{C}$ to provide protection from light.[7]

- Chemical peeling of the skin using glycolic acid or tricarboxylic acid. $[5,7]$

- Vitamin E and N-acetyl cysteine therapies to prevent damage to articular cartilage.[4]

- Antalgics (pain-reducers), anti-inflammatory medications used to treat arthritic symptoms.[7]

- Nitisinone, a potent inhibitor of an enzyme in the tyrosine catabolic pathway. It significantly lowers the urinary excretion of homogentisic acid by inhibiting 4-hydrophenylpyruvate dioxygenase and theoretically would reduce homogentisic acid accumulation. $[4,1]$

\section{B. Surgical Care}

- Knee, hip, shoulder and other joint replacements may be needed if ochronotic arthropathy progresses substantially.[4]

\section{Prevention}

Currently, there are no specific methods or guidelines to prevent ochronosis, since it is a genetic condition.

- Genetic testing of the expecting parents (and related family members) and prenatal diagnosis (Molecular testing of the foetus during pregnancy) may help in understanding the risks better during pregnancy. ${ }^{[7]}$

- If there is a family history of the condition, then genetic counselling will help assess risks before planning for a child.[7]
- Regular medical screening at periodic intervals with tests, scans and physical examinations are recommended.[7]

\section{FINAL DIAGNOSIS}

The diagnosis in this case is ochronotic joint disease with secondary involvement in the skin and the conjunctiva. Review of the literature suggests that ochronosis commonly affects all connective tissue and includes early changes in the upper extremity and small joints as well as decreased integrity of tendons, ligaments and muscles. Recognition of the changes secondary to deposition of ochronotic pigments has increased with advances in diagnostic technology allowing both improved imaging and early biochemical and genetics-based diagnosis of alkaptonuria. Nitisinone is the first pharmacotherapeutic agent that seems to prevent ochronosis, though further studies are required to assess its efficacy and possible side effects.

\section{REFERENCES}

[1] Keller JM, Macaulay W, Nercessian OA, et al. New developments in ochronosis: review of the literature. Rheumatology International 2005;25(2):81-5.

[2] Fisher AA, Davis MW. Alkaptonuric ochronosis with aortic valve and joint replacements and femoral fracture: a case report and literature review. Clinical Medicine \& Research 2004;2(4):209-15.

[3] Gürkanlar D, Daneyemez M, Solmaz I, et al. Ochronosis and lumbar disc herniation. Acta Neurochirurgica (Wien) 2006;148(8):891-4.

[4] https://emedicine.medscape.com/article/1104184.

[5] http://www.ncbi.nlm.nih.gov/pmc/articles/PMC4681 189/

[6] https://www.ncbi.nlm.nih.gov/pmc/articles/PMC348 2806/

[7] https://www.dovemed.com/diseasesconditions/ochronosis/ 